\title{
Utilização de polpa de frutas em pó \\ carregadoras de probióticos como alimento funcional: aspectos gerais e perspectivas
}

\section{Utilization of powdered fruit pulp probiotic loaders as a functional food: general aspects and perspectives}

\author{
Aline Pacheco Albuquerque ${ }^{1 *}$ (D), Thais Jaciane Araújo Rodrigues ${ }^{1}$, \\ Jonas Leite Cavalcante Neto $^{2}$, Ana Paula Trindade Rocha ${ }^{3}$ \\ ${ }^{1}$ Universidade Federal de Campina Grande (UFCG), Unidade Acadêmica de Engenharia Agrícola, Campina \\ Grande/PB - Brasil \\ ${ }^{2}$ Universidade Federal de Campina Grande (UFCG), Unidade Acadêmica de Engenharia de Alimentos, Campina \\ Grande/PB - Brasil \\ ${ }^{3}$ Universidade Federal de Campina Grande (UFCG), Unidade Acadêmica de Engenharia de Alimentos, Centro de \\ Tecnologia e Recursos Naturais, Campina Grande/PB - Brasil
}

*Corresponding Author: Aline Pacheco Albuquerque, Universidade Federal de Campina Grande (UFCG), Unidade Acadêmica de Engenharia Agrícola, R. Aprígio Veloso, 882, Universitário, CEP: 58429-900, Campina Grande/PB - Brasil, e-mail: aline.pacheco.qi@gmail.com

Cite as: Albuquerque, A. P., Rodrigues, T. J. A., Cavalcante Neto, J. L., \& Rocha, A. P. T. (2021). Utilization of powdered fruit pulp probiotic loaders as a functional food: general aspects and perspectives. Brazilian Journal of Food Technology, 24, e2019310. https://doi.org/10.1590/1981-6723.31019

\begin{abstract}
Resumo
O emprego de novos processos que possam agregar valor e aumentar a vida útil de produtos gerados a partir de frutas, bem como a preocupação com uma alimentação mais saudável, vem despertando um perfil mais inovador no mercado de alimentos. Dentre os mais diversos tipos de alimentos funcionais, estudos recentes vêm sendo desenvolvidos destacando os probióticos. Tradicionalmente, na elaboração de alimentos probióticos, são utilizados produtos derivados de leite, porém, atualmente, há um aumento na demanda por produtos não lácteos, devido ao crescimento do número de consumidores veganos, intolerantes à lactose e alérgicos às proteínas do leite. $O$ desenvolvimento de probióticos em novas matrizes vem se tornando uma opção cada vez mais atrativa para a indústria alimentícia. Logo, no presente artigo de revisão, são abordados os aspectos tecnológicos utilizados para análise da viabilidade de probióticos em polpas de frutas, as quais, por possuírem alto teor de umidade, são altamente perecíveis, necessitando da utilização de uma técnica de desidratação, com o intuito de reduzir as perdas pós-colheita, proteger contra as reações de degradação, contribuir para a concentração de nutrientes, além de possibilitar sua disponibilidade em qualquer período do ano. Assim, aspectos gerais da secagem em leito de jorro são discutidos como possível processo de conservação da polpa de frutas enriquecidas com culturas probióticas.
\end{abstract}

Palavras-chave: Bifidobactérias; Leito de jorro; Matriz vegetal; Produtos não lácteos; Secagem; Viabilidade microbiana. 


\begin{abstract}
The new processes to add value and increase the shelf life of products generated from fruits, as well as the concern with a healthier diet, have aroused a more innovative profile to the food market. Among the most diverse types of functional foods, probiotics have been highlighted by many recent studies. Traditionally, dairy products are used in the production of probiotic foods, but nowadays the demand for nondairy products has increased due to the growing number of consumers that are vegan, lactose intolerant and/or allergic to milk proteins. The development of probiotics in new matrices has become an increasingly attractive option for the food industry. Therefore, this review article discusses the technological aspects used to analyze the viability of probiotics in fruit pulps. Due to their high moisture content, fruit pulps are highly perishable, requiring a dehydration technique to reduce postharvest losses, protect against degradation reactions, contribute to nutrient concentration, and enable their availability at any time of the year. Thus, general aspects of spouted bed drying are discussed as a possible preservation process of fruit pulp enriched with probiotic cultures.
\end{abstract}

Keywords: Bifidobacteria; Spouted bed; Vegetable matrix; Non-dairy products; Drying; Microbial viability.

\title{
1 Introdução
}

O Brasil é um dos países de grande destaque no cenário mundial no que diz respeito à biodiversidade, além de sua posição geográfica privilegiada tornar possível a possibilidade de cultivo das mais inúmeras espécies frutíferas (Santos et al., 2018).

O consumo de frutas apresenta elevada aceitabilidade, todavia esse consumo também está associado ao alto grau de perecibilidade. Assim, devido aos períodos de sazonalidade, torna-se necessária a aplicação de tecnologias que possam fornecer um melhor aproveitamento dessas matérias-primas. $\mathrm{O}$ emprego de novos processos que possam agregar valor e aumentar a vida útil de produtos gerados a partir de frutas, aliado à diversidade de pesquisas na área agroindustrial, vem despertando um perfil mais inovador no mercado de alimentos.

Atualmente, alimentos que trazem consigo mecanismos de defesa biológica, prevenção, tratamento ou retardo de doenças, além da nutrição, são ditos funcionais, destacando-se por serem similares a um alimento convencional, sendo consumido como parte habitual da dieta, mas com capacidade comprovada de promover benefícios fisiológicos com constatação clínica (Aguiar et al., 2019).

Dentre os mais diversos tipos de alimentos funcionais, destacam-se os probióticos, normalmente usados em laticínios, como objetivo de diversos estudos recentes relacionando seus benefícios à saúde (Carvalho et al., 2017; Gallina et al., 2019; Leite et al., 2018; Rolim et al., 2020; Kim et al., 2020; Zendeboodi et al., 2020; Zhou et al., 2020).

Segundo Kleerebezem et al. (2019), uma grande variedade de produtos que contêm probióticos são consumidos diariamente por milhões de pessoas. Os probióticos são definidos como microrganismos vivos que, quando administrados em quantidades adequadas, conferem benefícios à saúde do hospedeiro (Hill et al., 2014). Uma pesquisa desenvolvida por Panigrahi et al. (2017) afirma que, a partir de 2018, quase 2.000 estudos clínicos relataram uma variedade de benefícios à saúde associados aos probióticos, incluindo um estudo recente que mostrou que uma mistura de probióticos e prebióticos resultou em uma redução de $40 \%$ da sepse neonatal e da morte, entre crianças na zona rural da Índia.

Para que um alimento seja considerado probiótico, recomenda-se que ele contenha no mínimo de $10^{8} \mathrm{a}$ $10^{9} \mathrm{UFC} / \mathrm{g}$ de produto, sendo as bactérias do gênero Lactobacillus e Bifidobacterium as mais amplamente utilizadas para o consumo humano (Tripathi \& Giri, 2014). Mesmo com grande relevância em relação aos aspectos positivos à saúde, as bifidobactérias ainda possuem um uso reduzido quando comparado ao das bactérias láticas (Holkem et al., 2016). 
Tradicionalmente, na elaboração de alimentos probióticos, são utilizados produtos lácteos. Porém, atualmente, há um aumento na demanda por produtos que não possuam origem animal, devido ao crescimento do número de consumidores veganos, bem como aqueles intolerantes à lactose e alérgicos às proteínas do leite. A inclusão de probióticos em alimentos não lácteos vem se tornando uma opção cada vez mais atrativa para a indústria alimentícia (Perricone et al., 2015). Portanto, esses microrganismos já vêm sendo incorporados em diversos produtos, seja na forma de bebidas ou até mesmo como suplementos em cápsulas (Holkem et al., 2016). Para a microencapsulação de probióticos, métodos de secagem vêm sendo utilizados, como atomização, fluidização e liofilização (Dimitrellou et al., 2016).

Neste contexto, observa-se que os probióticos são incorporados em produtos lácteos, como iogurtes, leites fermentados, entre outros. Por se tratar de produtos que estão diretamete ligados ao bom funcionamento da microbiota presente na flora intestinal, sendo a mesma um complexo sistema de comunicação no organismo, é nítida a necessidade do desenvolvimento de sucos de frutas adicionados de probióticos, representando uma opção de diversificação de produtos para a indústria, com utilização de uma nova matriz vegetal (Hugo et al., 2016; Paim et al., 2016).

A incorporação da cultura probiótica na polpa de frutas consiste, em termos gerais, na metodologia descrita por Paim et al. (2016) com modificações. Primeiramente, ocorrem seleção, lavagem, sanitização e trituração dos frutos, seguidas da adaptação de fatores, como, por exemplo, ajuste de $\mathrm{pH}$. Recomenda-se um tratamento térmico em que se utiliza, geralmente, a pasteurização, para eliminação de outros microrganismos no meio de cultivo (polpa). Após processamento, há a adequação da temperatura ideal de inoculação da cultura (em câmara asséptica), seguida da incubação (estufa bacteriológica), com temperatura e período de tempo propícios para que haja o desenvolvimento e a viabilidade das células probióticas.

Por possuir alto teor de umidade, sendo consideradas como alimentos altamente perecíveis, as frutas e alguns produtos oriundos delas exigem métodos de conservação e processamento, como processos de secagem, com o intuito de reduzir as perdas pós-colheita, proteger contra as reações de degradação, contribuir para a concentração de nutrientes, além de possibilitar sua disponibilidade em qualquer período do ano (Jafari et al., 2017).

Dentre os processos de conservação, a secagem tem o intuito de prolongar a vida útil, proporcionando um produto de qualidade para o consumidor e buscando, assim, uma alternativa industrial para aumentar a preservação das polpas, a manutenção da viabilidade da bactéria probiótica e a qualidade das propriedades funcionais do produto obtido (Broeckx et al., 2016; Zaccardi et al., 2017).

Ao buscar na literatura, é possivel encontrar alguns estudos disponíveis envolvendo a produção de pós de frutas como alimentos funcionais probióticos utilizando, algumas vezes, a técnica de microencapsulação dos microrganismos, protegendo as culturas de condições adversas de estocagem, no entanto são escassas pesquisas utilizando a desidratação em leito de jorro (Paim et al., 2016; Barbosa et al., 2015). A escolha da explanação acerca da secagem em leito de jorro parte do pressuposto de que, a partir desse método, é possível obter um pó com granulometria fina e uniforme, utilizando tempos de contato curtos durante o processo, além de apresentar menor inativação pelo calor pelo fato de utilizar temperaturas de ar de secagem baixas, elucidando o interesse da utilização dessa técnica, já que se trata de um produto com compostos termolábeis (Ferreira et al., 2015; Broeckx et al., 2016).

Assim, visando a obtenção e preservação de polpas de frutas probióticas em pó, a secagem por meio do secador em leito de jorro torna-se uma excelente alternativa de perfil inovador, a qual, segundo Tontul \& Topuz (2017), possibilita a manutenção e a viabilidade dos microrganismos durante a estocagem por maiores períodos de armazenamento, reduzindo as condições adversas durante a vida de prateleira e garantindo a permanência das propriedades funcionais (Huang et al., 2016).

Devido à importância desse assunto, atualmente, para a área das ciências e suas tecnologias, neste artigo de revisão, serão abordados os aspectos tecnológicos utilizados no desenvolvimento de culturas probióticas 
em polpas de frutas submetidas ao processo de secagem em leito de jorro que permitem aumentar a sua viabilidade durante a fermentação, o processamento e a utilização nos produtos alimentícios.

\section{Revisão}

\subsection{Aspectos gerais dos alimentos funcionais}

O perfil alimentar dos brasileiros vem se alterando gradativamente, sendo este caracterizado pelo consumo deficiente de nutrientes necessários para o bom funcionamento do organismo. Esses maus hábitos estão diretamente ligados ao aumento de doenças crônicas e obesidade (Reis et al., 2016).

Para a grande maioria da sociedade, o papel dos alimentos é de fundamental importância para uma boa qualidade de vida. A função inicial dos alimentos como fonte de energia e crescimento vem se transformando num perfil mais biológico, por meio de estudos da ação dos componentes presentes nos mesmos. Por conseguinte, são de total interesse do consumidor, produtos novos, de fácil acesso e alto valor nutritivo. Com isso, para atender esse novo mercado de consumo, o setor da produção alimentícia vem investindo na elaboração de "alimentos funcionais".

O mercado de alimentos funcionais tem sido de grande destaque no cenário mundial e as tendências relacionadas aos mesmos vêm evoluindo e crescendo em diferentes proporções, de acordo com os países. Segundo Corbo et al. (2014), isto ocorre devido a diferenças sociodemográficas e socioculturais na percepção do consumidor e na aceitação de produtos funcionais.

Os alimentos funcionais surgiram no Japão, em meados dos anos 1980, referindo-se a alimentos que são capazes de apresentar benefícios fisiológicos e/ou redução de doenças, além das funções nutricionais básicas, sendo de suma importância a comprovação científica clínica da sua eficácia. Estando eles totalmente ligados ao estilo de vida, nota-se a necessidade de os alimentos funcionais englobarem fatores capazes de suprir o empobrecimento de nutrientes, podendo ser vistos como um escape para os consumidores que não possuem uma boa alimentação, seja por questões rotineiras da atualidade ou por hábitos corriqueiros (Santos Moraes et al., 2018).

De acordo com Carvalho et al. (2017), os alimentos tidos como funcionais são comumente classificados como aqueles que, além de suprirem as funções nutritivas básicas, quando consumidos como parte da dieta usual, podem demonstrar benefícios adicionais em uma ou mais funções do organismo, trazendo melhorias do estado de saúde e bem-estar, ou diminuindo o risco de doenças.

Arruda et al. (2017) afirmam que os alimentos funcionais podem ser classificados em função do próprio alimento ou da presença de componentes bioativos que estejam presentes na sua composição, como as fibras, os fitoquímicos, as vitaminas, os minerais essenciais, os peptídeos bioativos, os probióticos, as ervas, os ácidos graxos e o ômega 3. Esses alimentos possuem potencial para promover a saúde através de mecanismos não previstos da nutrição convencional, sendo efeitos que se restringem à promoção de saúde e não à cura de doenças. Entre os alimentos funcionais, os alimentos com microrganismos probióticos são de grande importância, podendo-se ainda destacar os prebióticos e simbióticos.

A produção de alimentos funcionais contendo bactérias probióticas é uma área que vem ganhando destaque na indústria alimentícia nos últimos anos. Os consumidores estão mais conscientes da relação entre boa alimentação e saúde, e por isso tem aumentado a procura por alimentos que, além de nutrir, proporcionem benefícios à saúde dos consumidores (Nosrati et al., 2014).

\subsection{Probióticos e suas principais características}

Estudos afirmam que os probióticos representam um alimento funcional arquetípico, definidos como microrganismos vivos que, quando administrados em quantidades adequadas, conferem um benefício à saúde 
do consumidor, ou seja, funcionam como um suplemento microbiano vivo, que afetam beneficamente a saúde, melhorando o equilíbrio microbiano intestinal (Hill et al., 2014; Vandenplas et al., 2015).

Múltiplos estudos descreveram, dentre seus benefícios para a saúde, principalmente quando estão em condições adequadas de concentração, o controle da microbiota intestinal. Enfatiza-se que manter o equilíbrio da mesma pode assegurar uma suplementação sistemática, resultando em fatores positivos relacionados a efeitos antagônicos, imunológicos e a resistência a patógenos, ou seja, ocorre a proliferação de bactérias benéficas, estabilização da microbiota intestinal após o uso de antibióticos, promoção da digestão da lactose em indivíduos intolerantes, estimulação do sistema imunológico, alívio da constipação e aumento da absorção de minerais e vitaminas, apresentando ainda propriedades antimutagênicas e anticancerígenas (Hugo et al., 2016; Huang et al., 2016; Carvalho et al., 2017).

Na literatura, estudos comprovaram que o mecanismo de ação dos probióticos está diretamente relacionado com a competição por nutrientes e sítios de adesão, e a produção de metabólitos antimicrobianos, sendo, assim, capazes de colonizar o intestino, sintetizando compostos que possuem influência direta na instalação e permanência de patógenos, sendo eles os ácidos orgânicos voláteis, as bacteriocinas e o peróxido de hidrogênio (Santos Moraes et al., 2018).

Porém, para que os efeitos benéficos ocorram, de acordo com a Agência Nacional de Vigilância Sanitária (ANVISA), a dose mínima diária da cultura probiótica considerada terapêutica é de $10^{8}$ a $10^{9}$ UFC (Unidades Formadoras de Colônia), o que corresponde ao consumo de $100 \mathrm{~g}$ de um produto que contenha $10^{6}$ a $10^{7} \mathrm{UFC} / \mathrm{g}$ ou $\mathrm{mL}$ (Agência Nacional de Vigilância Sanitária, 2013). Contudo, para atender às especificações de um alimento probiótico, a bactéria deve ser inócua, possuir viabilidade para suportar a estocagem e o transporte, tolerar o $\mathrm{pH}$ do suco gástrico e possuir propriedades de resistência a fagos e oxigênio (Melo et al., 2016).

Os produtos probióticos são divididos em dois tipos: produtos probióticos lácteos, como iogurte, queijo, sorvete, fórmula infantil, bebidas com soro e sobremesas leiteiras, e produtos probióticos não lácteos, como grãos, doces, diferentes bebidas, como suco e cerveja não alcoólica, alimentos para bebês e produtos à base de carne (Mohammad et al., 2016).

Os probióticos foram primeiramente adicionados ao iogurte, assim como aos outros produtos lácteos fermentados. No entanto, um aumento na procura por produtos probióticos não lácteos passou a vigorar devido a fatores ligados a intolerância e alergia a componentes do leite, bem como a pessoas que não ingerem produtos de origem animal, dentre outros (Perricone et al., 2015). Desta forma, passaram-se a desenvolver produtos probióticos de várias matrizes alimentares, incluindo frutas, vegetais, assim como suas misturas (Mohammad et al., 2016; Cardoso et al., 2015).

Pesquisas desenvolvidas constam que o uso de probióticos em sucos de frutas e vegetais pode ser uma boa alternativa para o grupo de pessoas com necessidades especiais, como descrito anteriormente (Mohammad et al., 2016). Os sucos de frutas melhoram amplamente a saúde e possuem uma grande quantidade de antioxidantes, vitaminas, minerais, fibras dietéticas e outras substâncias nutricionais úteis para o organismo humano (Pereira et al., 2011).

Quando empregados em alimentos com alegação de propriedade funcional, os microrganismos probióticos devem mostrar resistência às operações de processamento e viabilidade no decorrer do período de armazenamento do produto, de acordo com Souza et al. (2019), o que justifica a utilização de um processo de secagem que possibilita essas condições.

\subsection{Principais bactérias probióticas e viabilidade celular}

As bactérias classificadas como probióticas, segundo a Agência Nacional de Vigilância Sanitária (2019), são: Lactobacillus delbrueckii, grupo do Lactobacillus plantarum ou grupo do Lactobacillus casei, além de Bifidobacterium animalis e Bifidobacterium longum.

Contudo, as bactérias mais amplamente utilizadas em alimentos probióticos são pertencentes aos gêneros Bifidobacterium e Lactobacillus. Ambas fazem parte do organismo humano e são consideradas desejáveis, por possuírem propriedades funcionais quando utilizadas isoladamente ou em conjunto, além de haver a disponibilidade comercial, facilitando, consequentemente, o acesso às mesmas (Witschinski et al., 2018).

De acordo com Silva (2016), na indústria, para um desenvolvimento com viabilidade celular, deve-se atender a parâmetros que melhorem a sobrevivência dessas bactérias probióticas, tais como: faixas de $\mathrm{pH}$ e 
temperatura adequadas, embalagens com barreira de oxigênio ou atmosfera modificada, adição de ingredientes antioxidantes, como glucose oxidase, cisteína ou ácido ascórbico, além da função protetora exercida pela própria matriz alimentícia

Embora essas culturas probióticas possam apresentar grande variabilidade morfológica em relação às condições de crescimento, quantidade de metabólitos e respostas a nutrientes, as bifidobactérias são de interesse no viés da pesquisa, tendo em vista a dificuldade de encontrar informações sobre as variações das suas especificações de crescimento. Além disso, as bifidobactérias, assim como os lactobacilos, podem trazer diversos benefícios no controle de infecções intestinais, no tratamento relacionado à intolerância à lactose, na inativação de compostos tóxicos e no alívio de sintomas gastrointestinais, em mulheres com síndrome prémenstrual (Silva, 2016).

Tratando-se do armazenamento a baixas temperaturas, quando associado a produtos com uso dessas bactérias, como, por exemplo, no congelamento de polpas de frutas probióticas, observa-se um fator considerado negativo, em virtude dos possíveis danos que a temperatura pode oferecer aos microrganismos probióticos, ocasionando uma inviabilidade celular para as bactérias (Broeckx et al., 2016).

Com isso, é nítida a necessidade de associar um método de conservação e a junção da polpa de frutas com cultura probiótica, buscando assim uma alternativa para aumentar a preservação das polpas, a manutenção da viabilidade da bactéria probiótica e a qualidade das propriedades funcionais do produto obtido (Broeckx et al., 2016; Zaccardi et al., 2017).

\subsection{Polpa de frutas probióticas em pó}

O desenvolvimento de tecnologias acessíveis para produção de pós de frutas com características probióticas surge como alternativa de melhorar o aproveitamento da matéria-prima oriunda dessas espécies, com possibilidades de aumento de renda em toda a cadeia produtiva junto à agroindústria regional, ao disponibilizar um novo produto no segmento de frutas desidratadas com alegação funcional.

A indústria de alimentos em pó tem apresentado crescimento devido às vantagens que a utilização e o manejo dos materiais oferecem, principalmente, no que diz respeito ao seu emprego como matéria-prima ou aditivo, à facilidade de conservação em longo prazo e ao baixo custo com armazenamento (Moura et al., 2020).

$\mathrm{Na}$ literatura, estão disponíveis alguns estudos para produção de frutas probióticas em pó com uso de diferentes microrganismos e processos de secagem. Na Tabela 1, estão inseridos alguns trabalhos que foram desenvolvidos visando à obtenção de pós probióticos à base de frutas, acompanhada do método de secagem que foi utilizado e da cultura probiótica. Algumas pesquisas conciliam os probióticos e os prebióticos.

Tabela 1. Estudos referentes a elaboração de polpas de frutas em pó com utilização de probióticos.

\begin{tabular}{lccc}
\hline \multicolumn{1}{c}{ Referência } & Fruta & Método de secagem & Microrganismos \\
\hline Paim et al. (2016) & Jussara & Spray drying & Bifidobacterium spp. Lactis \\
\hline Barbosa et al. (2015) & Laranja & Spray drying & $\begin{array}{c}\text { Lactobacillus plantarum e Pediococcus } \\
\text { acidilactici }\end{array}$ \\
\hline Anekella \& Orsat (2013) & Framboesa & Spray drying & $\begin{array}{c}\text { Lactobacillus acidophilus } \text { e } \\
\text { Lactobacillus rhamnosus }\end{array}$ \\
\hline Lima (2013) & Banana & Liofilização & Lactobacillus acidophilus \\
\hline Pereira et al. (2011) & Caju & Spray drying & Lactobacillus casei \\
\hline Almeida (2012) & Abacaxi & Spray drying & Lactobacillus casei \\
\hline Rocha (2013) & Tangerina & Leito de jorro & Leuconostoc mesenteroides \\
\hline Mestry et al. (2011) & $\begin{array}{c}\text { Cenoura com } \\
\text { melancia }\end{array}$ & Spray drying & Lactobacillus acidophilus \\
\hline
\end{tabular}


Silva et al. (2015) salientam que um dos fatores que apresentam grande relevância na qualidade de pós de frutas é a atividade de água, devido ao fato de este parâmetro ser de fundamental importância para a conservação e o armazenamento de um produto, em razão de influenciar diretamente nas características de sua composição e na sua estabilidade.

De acordo com Mestry et al. (2011), o pó de suco fermentado é altamente desejável porque o produto seco potencialmente tem vida de prateleira mais longa e menor custo de transporte. Além disso, os pós probióticos de suco de frutas e vegetais podem ser usados em bebidas probióticas, sorvetes probióticos, xaropes, sopas preparadas, dentre outros.

Mediante explanação, pode ser observado que a existência de trabalhos utilizando o método de secagem em leito de jorro é escassa. Todavia, as técnicas desse método têm evoluído consideravelmente, ocupando importante lugar no processamento de alimentos substituindo inclusive o método de spray drying, em alguns casos.

\subsection{Método de secagem em leito de jorro}

A secagem é um dos métodos de conservação mais empregados atualmente para aumentar a qualidade de alimentos líquidos e pastosos, como polpa de frutas, tendo como principal intuito a obtenção de um produto em pó com elevado valor agregado.

Entre os métodos de secagem que surgiram, a técnica do leito de jorro foi desenvolvida, em 1974, pelos pesquisadores canadenses Mathur e Gislher, para a secagem de grãos. Nos anos seguintes, diversas pesquisas foram realizadas com o equipamento para uma variedade de materiais, ampliando o conhecimento e a aplicação dessa técnica em todo o mundo (Mathur \& Epstein, 1974; Epstein \& Grace, 2011).

No Brasil, a secagem de frutas tropicais utilizando os secadores de leito de jorro tem sido muito estudada, devido à excelente qualidade dos produtos em pó que estão sendo obtidos (Rocha et al., 2011).

A secagem por leito de jorro é considerada como um tipo de secagem de baixo custo e capaz de produzir produtos com qualidade semelhante à dos obtidos por outros métodos tradicionais de secagem. A secagem de pastas e suspensões em leito de jorro tem se mostrado uma alternativa à secagem em spray drying. Uma das principais vantagens da secagem por leito de jorro é o fato de se poder trabalhar a baixas temperaturas, quando comparada com outras técnicas de secagem (Fujita et al., 2013). Essas temperaturas podem contribuir para a sobrevivência do microrganismo probiótico durante e após a secagem, já que as altas temperaturas utilizadas durante a tradicional secagem por spray drying são a principal causa da perda de viabilidade de culturas probióticas (Golowczyc et al., 2011).

Dessa forma, a secagem em leito de jorro é vista como um processo que promove a evaporação da água presente no alimento, conservando o teor de vitaminas e nutrientes do mesmo, produzindo assim um pó com estabilidade e preservação das características sensoriais do produto in natura (Pontes Júnior et al., 2015).

Diversos estudos têm evidenciado que a utilização do secador tipo leito de jorro diferencia-se das demais devido às suas principais características, sendo estas: capacidade de proporcionar excelente grau de mistura e eficiente contato entre o fluido e o material sólido, alta taxa de circulação de partículas inertes, bons coeficientes de transferência de calor e massa, e a uniformidade da temperatura no leito, tudo em tempos de contato curtos, contribuindo desta maneira para a qualidade do produto (Santos Melo et al., 2010). De acordo com Ferreira et al. (2019), a secagem utilizando o leito de jorro permite que se obtenha um pó com granulometria fina e uniforme.

$\mathrm{Na}$ forma original e convencional, o leito de jorro é constituído basicamente por uma coluna cilíndrica e uma base cônica, na qual se localiza a entrada do fluido que irá escoar pelo material particulado, que é, na maioria das vezes, o ar. O jorro é formado quando a vazão de ar é suficiente para provocar o movimento ascendente das partículas acima do leito, podendo ser observado por regiões: região central ou canal de jorro, caracterizada pelo movimento ascendente das partículas; região da fonte, denotada como a região acima da 
superfície do leito; região anular, que apresenta comportamento semelhante a um leito deslizante. $\mathrm{O}$ movimento completo realizado é estabelecido como um movimento cíclico e sistemático, capaz de proporcionar uma mistura eficaz do material particulado e um elevado grau de contato entre as fases fluida e sólida (Sousa, 2017).

Para garantir total eficiência do processo, devem-se manter valores altos de coeficientes de transferência de calor e massa, ampla área de contato, fornecimento elevado de calor pelo gás de secagem, temperatura invariável ao longo da câmara de secagem, injeção de suspensões concentradas para diminuir a quantidade de água a ser evaporada e emprego de ar com temperaturas elevadas na entrada (Machado et al., 2015).

No entanto, materiais ricos em açúcares e ácidos de baixo peso molecular, como as polpas de frutas, produzem pós muito higroscópicos, suscetíveis à aglomeração e com problemas de fluidez. Contudo, este problema pode ser evitado ou, pelo menos, minimizado com a adição dos adjuvantes de secagem, que são carboidratos de alto peso molecular, como as maltodextrinas, que reduzem a higroscopicidade dos pós e facilitam o processo de secagem (Freitas et al., 2019).

\subsection{Adjuvantes de secagem}

A problemática de aglomeração devido à higroscopicidade nas polpas de frutas em pó resulta dos açúcares de baixo peso molecular, que possuem baixa temperatura de transição vítrea, tornando-se gomosos quando entram em contato com temperaturas elevadas. Para solução desse problema, é necessário o uso de carboidratos de alto peso molecular que contêm alta temperatura de transição vítrea e podem assim reduzir a higroscopicidade dos pós, facilitando o processo de secagem (Torres et al., 2016).

De acordo com Goula \& Adamopoulos (2010), comumente são utilizados como aditivos de secagem substâncias inertes, em geral de alto peso molecular, como proteínas e maltodextrina com diferentes dextroses equivalentes, sendo esses agentes capazes de estabilizar a temperatura de transição vítrea e, consequentemente, evitar problemas, como a aglomeração, a pegajosidade e o colapso.

Diversos produtos além de amidos e dextrinas podem ser utilizados como adjuntos de secagem de polpas de frutas por atomização. Entre os aditivos, podemos citar: amido, dextrinas, açúcar, xarope de milho, celuloses, goma arábica, alginato de sódio, carragena, monoglicerídeos e diglicerídeos, óleos e gorduras hidrogenadas, poliésteres naturais, polímeros sintéticos, glúten, caseína, gelatina, albumina e quitosana. A maltodextrina é um dos aditivos mais utilizados, pois, além do baixo custo, apresenta baixa higroscopicidade, evita a aglomeração das partículas, possui efeito antioxidante e retém os voláteis na faixa de 65 a $80 \%$. Já os frutanos, como inulina e oligofrutose, não são habitualmente usados, mas são reconhecidos como uma classe de carboidratos que, depois do amido, são os polissacarídeos não estruturais de maior ocorrência entre as plantas, possuindo alto teor de polimerização (Paim et al., 2016).

Com base na diversificação dos adjuvantes de secagem, é importante evidenciar que a escolha de qual adjuvante usar está diretamente relacionada com a sua composição, pois essas características constituem suas propriedades funcionais e de como ele pode ser utilizado para melhorar o desempenho durante os processos de secagem, permitindo um controle sofisticado de certas propriedades do produto.

\section{Conclusão}

Conclui-se que diversos estudos têm demostrado que a utilização de polpa de frutas na elaboração de produtos probióticos em pó é viável como fonte de nutrientes para o desenvolvimento e a manutenção de células probióticas numa nova matriz de origem vegetal, em substituição de produtos oriundos de leite.

A utilização da técnica de secagem em leito de jorro torna-se uma alternativa atrativa quando estima-se um produto que conserve suas principais características sensoriais e nutricionais, garantindo a obtenção de um alimento funcional com granulometria uniforme, de fácil transporte e maior tempo de armazenamento. 


\section{Referências}

Agência Nacional de Vigilância Sanitária - ANVISA. (2013). Alimentos com alegação de propriedades funcionais e ou de saúde, novos alimentos/ingredientes, substâncias bioativas e probióticos. Brasília.

Agência Nacional de Vigilância Sanitária - ANVISA. (2019). Guia para instrução processual de petição de avaliação de probióticos para uso em alimentos. Brasília.

Aguiar, L. M., Geraldi, M. V., Cazarin, C. B. B., \& Maróstica Junior, M. R. (2019). Functional food consumption and its physiological effects. In M. R. S. Campos, editor. Bioactive compounds: Health benefits and potential applications (pp. 205-225). Duxford: Woodhead Publishing. http://dx.doi.org/10.1016/B978-0-12-814774-0.00011-6.

Almeida, F. D. L. (2012). Desidratação de suco de abacaxi probiótico por spray dryer (Dissertação de mestrado). Universidade Federal do Ceará, Fortaleza.

Anekella, K., \& Orsat, V. (2013). Optimization of microencapsulation of probiotics in raspberry juice by spray drying. Lebensmittel-Wissenschaft + Technologie, 50(1), 17-24. http://dx.doi.org/10.1016/j.Iwt.2012.08.003

Arruda, É. F., Oliveira, A., \& Oliveira, A. D. (2017). Avaliação de sorvete tipo iogurte à base de soja com a adição de microrganismos probióticos. Episteme Transversalis, 6(2), 1-23.

Barbosa, J., Borges, S., Amorim, M., Pereira, M. J., Oliveira, A., Pintado, M. E., \& Teixeira, P. (2015). Comparison of spray drying, freeze drying and convective hot air drying for the production of a probiotic orange powder. Journal of Functional Foods, 17, 340-351. http://dx.doi.org/10.1016/j.jff.2015.06.001

Broeckx, G., Vandenheuvel, D., Claes, I. J., Lebeer, S., \& Kiekens, F. (2016). Drying techniques of probiotic bacteria as an important step towards the development of novel pharmabiotics. International Journal of Pharmaceutics, 505(1-2), 303-318. PMid:27050865. http://dx.doi.org/10.1016/j.ijpharm.2016.04.002

Cardoso, B. V. S., Freire, J. A. P., Oliveira, G. A. L., Sousa, I. G. M., Freitas, R. M., \& Nunes, L. C. C. (2015). Prospecção tecnológica e científica de alimentos probióticos funcionais na forma de barra de cereais. Revista GEINTEC: Gestão, Inovação e Tecnologias, 5(3), 2273-2283.

Carvalho, F. L. O., Uyeda, M., del Buonom, H. C., \& Gonzaga, M. F. N. (2017). Probióticos e prebióticos: Benefícios acerca da literatura. Revista de Saúde ReAGES, 1(1), 58-87.

Corbo, M. R., Bevilacqua, A., Petruzzi, L., Casanova, F. P., \& Sinigaglia, M. (2014). Functional beverages: The emerging side of functional foods: Commercial trends, research, and health implications. Comprehensive Reviews in Food Science and Food Safety, 13(6), 1192-1206. http://dx.doi.org/10.1111/1541-4337.12109

Dimitrellou, D., Kandylis, P., Petrović, T., Dimitrijević-Branković, S., Lević, S., Nedović, V., \& Kourkoutas, Y. (2016). Survival of spray dried microencapsulated Lactobacillus casei ATCC 393 in simulated gastrointestinal conditions and fermented milk. Lebensmittel-Wissenschaft + Technologie, 71, 169-174. http://dx.doi.org/10.1016/j.Iwt.2016.03.007

Epstein, N., \& Grace, J. R. (2011). Spouted and spout-fluid beds: Fundamentals and applications. New York: Cambridge University Press.

Ferreira, A., Sousa, S., Lima, D., \& Costa, J. (2015). Caracterização de polpa de cajá em pó obtida pelo método de secagem em leito de jorro. Blucher Chemical Engineering Proceedings, 1(2), 3997-4004. http://dx.doi.org/10.5151/chemeng-cobeq20140807-23642-180446

Ferreira, S., Araujo, T., Souza, N., Rodrigues, L., Lisboa, H. M., Pasquali, M., Trindade, G., \& Rocha, A. P. (2019).

Physicochemical, morphological and antioxidant properties of spray-dried mango Kernel starch. Journal of Agriculture and Food Research, 1, 100012. http://dx.doi.org/10.1016/j.jafr.2019.100012

Freitas, E. D. F. M., Lopes, L. D. L., Alves, S. M. D. F., \& Campos, A. J. D. (2019). Efeito da maltodextrina no sumo da polpa de abacaxi Pérola atomizado. Revista de Ciências Agrárias, 42(1), 271-280.

Fujita, A., Borges, K., Correia, R., Melo Franco, B. D. G., \& Genovese, M. I. (2013). Impact of spouted bed drying on bioactive compounds, antimicrobial and antioxidant activities of commercial frozen pulp of camu-camu (Myrciaria dubia Mc. Vaugh). Food Research International, 54(1), 495-500. http://dx.doi.org/10.1016/j.foodres.2013.07.025

Gallina, D. A., Ormenese, R. C. S. C., \& Garcia, A. O. (2019). logurte probiótico com polpa de frutas vermelhas: Caracterização físico química e microbiológica, aceitabilidade sensorial e viabilidade dos probióticos. Revista do Instituto de Latícinios Cândido Tostes, 73(4), 196-208. http://dx.doi.org/10.14295/2238-6416.v73i4.681

Golowczyc, M. A., Silva, J., Teixeira, P., De Antoni, G. L., \& Abraham, A. G. (2011). Cellular injuries of spray-dried Lactobacillus spp. isolated from kefir and their impact on probiotic properties. International Journal of Food Microbiology, 144(3), 556-560. PMid:21144610. http://dx.doi.org/10.1016/j.ijfoodmicro.2010.11.005

Goula, A. M., \& Adamopoulos, K. G. (2010). A new technique for spray drying orange juice concentrate. Innovative Food Science \& Emerging Technologies, 11(2), 342-351. http://dx.doi.org/10.1016/j.ifset.2009.12.001

Hill, C., Guarner, F., Reid, G., Gibson, G. R., Merenstein, D. J., Pot, B., Morelli, L., Canani, R. B., Flint, H. J., Salminen, S., Calder, P. C., \& Sanders, M. E. (2014). The International Scientific Association for Probiotics and Prebiotics consensus statement on the scope and appropriate use of the term probiotic. Nature Reviews. Gastroenterology \& Hepatology, 11(8), 506514. PMid:24912386. http://dx.doi.org/10.1038/nrgastro.2014.66

Holkem, A. T., Raddatz, G. C., Nunes, G. L., Cichoski, A. J., Jacob-Lopes, E., Grosso, C. R. F., \& Menezes, C. R. (2016). Development and characterization of alginate microcapsules containing Bifidobacterium BB-12 produced by emulsification/internal gelation followed by freeze drying. Lebensmittel-Wissenschaft + Technologie, 71, 302-308. http://dx.doi.org/10.1016/j.Iwt.2016.04.012 
Huang, S., Cauty, C., Dolivet, A., Le Loir, Y., Chen, X. D., Schuck, P., Jan, G., \& Jeantet, R. (2016). Double use of highly concentrated sweet whey to improve the biomass production and viability of spray-dried probiotic bacteria. Journal of Functional Foods, 23, 453-463. http://dx.doi.org/10.1016/j.jf.2016.02.050

Hugo, A. A., Bruno, F., \& Golowczyc, M. A. (2016). Whey permeate containing galacto-oligosaccharides as a medium for biomass production and spray drying of Lactobacillus plantarum CIDCA 83114. Lebensmittel-Wissenschaft + Technologie, 69, 185-190. http://dx.doi.org/10.1016/j.Iwt.2016.01.031

Jafari, S. M., Ghalenoei, M. G., \& Dehnad, D. (2017). Influence of spray drying on water solubility index, apparent density, and anthocyanin content of pomegranate juice powder. Powder Technology, 311, 59-65. http://dx.doi.org/10.1016/j.powtec.2017.01.070

Kim, J. Y., Lee, S. Y., Jung, S. H., Kim, M. R., Choi, I. D., Lee, J. L., Sim, J. H., Pan, C. H., \& Kang, K. (2020). Protective effect of Lactobacillus casei HY2782 against particulate matter toxicity in human intestinal CCD-18Co cells and Caenorhabditis elegans. Biotechnology Letters, 42(4), 519-528. PMid:31970557. http://dx.doi.org/10.1007/s10529-020-02814-3

Kleerebezem, M., Binda, S., Bron, P. A., Gross, G., Hill, C., van Hylckama Vlieg, J. E., Lebeer, S., Satokari, R., \& Ouwehand, A. C. (2019). Understanding mode of action can drive the translational pipeline towards more reliable health benefits for probiotics. Current Opinion in Biotechnology, 56, 55-60. PMid:30296737. http://dx.doi.org/10.1016/j.copbio.2018.09.007

Leite, S. T., Roberto, C. D., Silva, P. I., \& Carvalho, R. V. (2018). Polpa de juçara: Fonte de compostos fenólicos, aumento da atividade antioxidante e da viabilidade de bactérias probióticas de iogurte. Revista Ceres, 65(1), 16-23. http://dx.doi.org/10.1590/0034-737x201865010003

Lima, D. C. N. (2013). Suco de banana em pó probiótico (Dissertação de mestrado). Universidade Estadual de Campinas, Campinas.

Machado, I., Ferreira, A. D. S., Machado, A., Delmiro, T., \& Medeiros, M. D. F. (2015). Secagem em leito de jorro da polpa de graviola com adição de leite: Efeito das variáveis de operação sobre a taxa de produção e umidade do pó. Blucher Chemical Engineering Proceedings, 1(2), 5902-5909. http://dx.doi.org/10.5151/chemeng-cobeq2014-1352-19691-174035

Mathur, K. B., \& Epstein, N. (1974). Spouted beds. New York: Academic Press.

Melo, T. A., Ribeiro-Alves, M. A., Lavinas, F. C., \& Almeida Rodrigues, I. (2016). Levantamento e caracterização dos produtos probióticos disponíveis no mercado varejista da região metropolitana do Rio de Janeiro. Revista Rede de Cuidados em Saúde, 10(1), 1-3.

Mestry, A. P., Mujumdar, A. S., \& Thorat, B. N. (2011). Optimization of spray drying of an innovative functional food: Fermented mixed juice of carrot and watermelon. Drying Technology, 29(10), 1121-1131. http://dx.doi.org/10.1080/07373937.2011.566968

Mohammad, M. Z., Mahnaz, H., \& Shila, B. (2016). Production of probiotic fermented mixture of carrot, beet and apple juices. Journal of Paramedical Sciences, 7(3), 17-23.

Moura, L. S., Anciens, G. L. L., \& Azeredo, D. R. (2020). Percepção dos consumidores sobre os riscos em alimentos. Alimentos: Ciência, Tecnologia e Meio Ambiente, 1(3), 16-31.

Nosrati, R., Hashemiravan, M., \& Talebi, M. (2014). Fermentation of vegetables juice by probiotic bacteria. International Journal of Biosciences, 4, 171-180.

Paim, D. R., Costa, S. D., Walter, E. H., \& Tonon, R. V. (2016). Microencapsulation of probiotic jussara (Euterpe edulis M.) juice by spray drying. $L W T, 74,21-25$. http://dx.doi.org/10.1016/j.Iwt.2016.07.022

Panigrahi, P., Parida, S., Nanda, N. C., Satpathy, R., Pradhan, L., Chandel, D. S., Baccaglini, L., Mohapatra, A., Mohapatra, S. S., Misra, P. R., Chaudhry, R., Chen, H. H., Johnson, J. A., Morris, J. G., Paneth, N., \& Gewolb, I. H. (2017). A randomized synbiotic trial to prevent sepsis among infants in rural India. Nature, 548(7668), 407-412. PMid:28813414. http://dx.doi.org/10.1038/nature23480

Pereira, A. L. F., Maciel, T. C., \& Rodrigues, S. (2011). Probiotic beverage from cashew apple juice fermented with Lactobacillus casei. Food Research International, 44(5), 1276-1283. http://dx.doi.org/10.1016/j.foodres.2010.11.035

Perricone, M., Bevilacqua, A., Altieri, C., Sinigaglia, M., \& Corbo, M. R. (2015). Challenges for the production of probiotic fruit juices. Beverages, 1(2), 95-103. http://dx.doi.org/10.3390/beverages1020095

Pontes Júnior, S. M., Dantas, S. C. M., Delmiro, T. M., \& Medeiros, M. F. D. (2015). Secagem de polpas de frutas em leito de jorro: Efeitos da adição de leites vegetal e animal e da proteína isolada de leite no desempenho do processo e qualidade do produto em pó produzido. In Anais do XI Congresso Brasileiro de Engenharia Química em Iniciação Científica (pp. 2069-2074). São Paulo: Blucher.

Reis, A. R. R., Soares, J. M. D., Souza, A. G. D., \& Messias, C. M. B. D. O. (2016). Conhecendo os benefícios dos alimentos: Alimentos funcionais. EXTRAMUROS-Revista de Extensão da Univasf, 4(2), 124-136.

Rocha, S. A. S. D. (2013). Obtenção de suco em pó prebiótico de tangerina através de secagem em leito de jorro (Dissertação de mestrado). Universidade Federal do Ceará, Fortaleza.

Rocha, S. C. S., Souza, J. S., Alsina, O. L. S., \& Medeiros, M. F. D. (2011). Drying of tropical fruit pulps: Spouted bed process optimization as a function of pulp composition. Drying Technology, 29(13), 1587-1599. http://dx.doi.org/10.1080/07373937.2011.585442

Rolim, F. R., Freitas Neto, O. C., Oliveira, M. E. G., Oliveira, C. J., \& Queiroga, R. C. (2020). Cheeses as food matrixes for probiotics: In vitro and in vivo tests. Trends in Food Science \& Technology, 100, 138-154. http://dx.doi.org/10.1016/j.tifs.2020.04.008

Santos Melo, K., Nascimento, M. A., Gomes, W. C., Cabral, S. B., \& Rocha, A. P. T. (2010). Fluidodinâmica de leito de jorro com leite de cabra e polpa de cajá. Revista Verde de Agroecologia e Desenvolvimento Sustentável, 5(4), 61-67. 
Santos Moraes, M., Santos Oliveira, L. P., de Carvalho Furtado, C., \& Gonzalez, F. G. (2018). Efeitos funcionais dos probióticos com ênfase na atuação do kefir no tratamento da disbiose intestinal. UNILUS Ensino e Pesquisa, 14(37), 144-156.

Santos, E. F., Araújo, R. R., Lemos, E. E. P., \& Endres, L. (2018). Quantificação de compostos bioativos em frutos de umbu (Spondias tuberosa Arr. Câm.) e Cajá (Spondias mombin L.) nativos de Alagoas. Revista de Ciencias Agricolas, 16(1), 21-29.

Silva, R. N., Figueirêdo, R. D., Queiroz, A. D. M., \& Feitosa, R. M. (2015). Isotermas de adsorção de umidade do umbu-cajá em pó. Revista Educação Agrícola Superior, 30(1), 33-36. http://dx.doi.org/10.12722/0101-756X.v30n01a07

Silva, S. F. (2016). Avaliação de sistemas de embalagem na estabilidade do queijo Minas Frescal probiótico e na viabilidade da Bifidobacterium animalis subsp. lactis (Tese de doutorado). Universidade Estadual de Campinas, Campinas.

Sousa, R. C. D. (2017). Secagem de materiais pastosos e granulares no leito de jorro mecânico e convencional (Tese de doutorado). Universidade Federal de São Carlos, São Carlos.

Souza, B. V. C., Rocha, P. O., Brito, C. A. R. S., Galvão, L. M. V., \& Nunes, L. C. C. (2019). Scientific and technological prospection on microencapsulation of probiotics by spray drying. Revista GEINTEC-Gestão. Inovação e Tecnologias, 9(2), 4919-4928.

Tontul, I., \& Topuz, A. (2017). Spray-drying of fruit and vegetable juices: Effect of drying conditions on the product yield and physical properties. Trends in Food Science \& Technology, 63, 91-102. http://dx.doi.org/10.1016/j.tifs.2017.03.009

Torres, J. K. F., Stephani, R., Tavares, G. M., Carvalho, A. F., Costa, R. G. B., Schuck, P., \& Perrone, Í. T. (2016). Hidrólise da lactose e produção de leite em pó: Aspectos tecnológicos. Revista do Instituto de Latícinios Cândido Tostes, 71(2), 94-105. http://dx.doi.org/10.14295/2238-6416.v70i2.529

Tripathi, M. K., \& Giri, S. K. (2014). Probiotic functional foods: Survival of probiotics during processing and storage. Journal of Functional Foods, 9, 225-241. http://dx.doi.org/10.1016/j.jff.2014.04.030

Vandenplas, Y., Huys, G., \& Daube, G. (2015). Probiotics: An update. Jornal de Pediatria, 91(1), 6-21. PMid:25458874. http://dx.doi.org/10.1016/j.jped.2014.08.005

Witschinski, F., Demartini, D., Kilian, J., Dallago, R. M., Rosa, C. D., Cansian, R. L., Valduga, E., \& Steffens, J. (2018). Development and characterization of light yoghurt elaborated with Bifidobacterium animalis subsp. Ciência Rural, 48(3), http://dx.doi.org/10.1590/0103-8478cr20170560

Zaccardi, H., Castro, J., Müller, T., Rocha, S., \& Pinto, L. (2017). Influência da temperatura de secagem em leito de jorro de pasta de vegetais enriquecida de plasma bovino nas características do produto em pó. Blucher Chemical Engineering Proceedings, 1(4), 1731-1736. http://dx.doi.org/10.5151/chemeng-cobeqic2017-306

Zendeboodi, F., Khorshidian, N., Mortazavian, A. M., \& Cruz, A. G. (2020). Probiotic: Conceptualization from a new approach. Current Opinion in Food Science, 32, 103-123. http://dx.doi.org/10.1016/j.cofs.2020.03.009

Zhou, Z., Chen, X., Sheng, H., Shen, X., Sun, X., Yan, Y., Wang, J., \& Yuan, Q. (2020). Engineering probiotics as living diagnostics and therapeutics for improving human health. Microbial Cell Factories, 19(1), 56. PMid:32131831.

http://dx.doi.org/10.1186/s12934-020-01318-z 